\title{
Dexmedetomidine Alleviates Lipopolysaccharide- Induced hippocampal neuronal apoptosis via inhibiting the p38 MAPK/c-Myc/CLIC4 Signaling Pathway in Rats
}

\author{
Yongping Chen \\ Northeast Agricultural University \\ Lin Li \\ Northeast Agricultural University \\ Jiuyan Zhang \\ Northeast Agricultural University \\ Hailin Cui \\ Northeast Agricultural University \\ Jiucheng Wang \\ Northeast Agricultural University \\ Chuqiao Wang \\ Northeast Agricultural University \\ Mingxian Shi \\ Northeast Agricultural University \\ Honggang Fan ( $\nabla$ fanhonggang2002@163.com ) \\ Northeast Agricultural University https://orcid.org/0000-0002-7671-6908
}

\section{Research Article}

Keywords: Dexmedetomidine, Lipopolysaccharide, Hippocampal neuronal apoptosis, p38 MAPK/cMyc/CLIC4 signaling pathway, Alzheimer's disease, Depression

Posted Date: June 2nd, 2021

DOI: https://doi.org/10.21203/rs.3.rs-538490/v1

License: (c) (1) This work is licensed under a Creative Commons Attribution 4.0 International License. Read Full License 
Version of Record: A version of this preprint was published at Molecular Neurobiology on August 7th, 2021. See the published version at https://doi.org/10.1007/s12035-021-02512-9. 


\section{Abstract}

Dexmedetomidine (DEX) reportedly possessed multiple bioactivities. Here, we mainly investigated the neuroprotective role and detailed molecular mechanism of DEX against lipopolysaccharide (LPS)-induced hippocampal neurons apoptosis. In vivo, Sprague Dawley rats were administered with LPS $(10 \mathrm{mg} / \mathrm{kg})$ and/or DEX $(30 \mu \mathrm{g} / \mathrm{kg})$. We found that DEX improved LPS-induced hippocampal microstructure (necrosis and number reduction of neurons in the CA1 and CA3 regions) and ultrastructure (mitochondrial damage) lesions. DEX also attenuated LPS-induced hippocampal apoptosis by down-regulating the expression of mitochondrial apoptosis pathway-related proteins. Moreover, DEX prevented the activation of cMyc/chloride intracellular channel 4 (CLIC4) pathway induced by LPS. Notably, DEX inhibited p38 MAPK pathway, not JNK and ERK. To further clarify whether DEX alleviated LPS-induced neuronal apoptosis through the p38 MAPK/c-Myc/CLIC4 pathway, PC12 cells were treated with p38 MAPK inhibitor SB203582 $(10 \mu \mathrm{M})$. As expected, DEX had the same effect as SB203582 in reducing the protein and mRNA expression of c-Myc and CLIC4. Furthermore, DEX and SB203582 diminished LPS-induced apoptosis, showing decreased Bax and Tom20 fluorescent double-stained positive cells, reduced Annexin V-FITC/PI apoptosis rate, and lessened protein expression levels of Bax, cytochrome C, cleaved caspase-9 and cleaved caspase-3. Taken together, DEX attenuates LPS-induced hippocampal neuronal apoptosis by regulating the $\mathrm{p} 38 \mathrm{MAPK} / \mathrm{c}-\mathrm{Myc} / \mathrm{CLIC} 4$ signaling pathway, which will provide new insights into the mechanism research and drug development of Alzheimer's disease and depression.

\section{Introduction}

Alzheimer's disease (AD) and depression pose a huge threat to human health and are major causes of increasing morbidity and mortality worldwide. According to incomplete statistics, approximately, 5.4 million people in the United States alone have AD [1]. Beyond that, more than 350 million victims in the world are currently affected by depression [2], which is predicted to be the world's second largest disability-inducing factor by 2030 [3]. Notably, hippocampal injury (loss of neuronal populations [4], reduced hippocampal volume [5], and massive neuronal death [6]) is regarded as an important pathological hallmark of $A D$ and depression. Numerous studies have corroborated that apoptosis is implicated in hippocampal injury. Clearly, it is necessary to find safe, side-effect-free antiapoptotic drugs to prevent and treat $A D$ and depression.

Lipopolysaccharide (LPS) was found to be abundant in hippocampal lysates of AD brain [7]. It is well known that LPS can give rise to depression [8]. Li et al. revealed that LPS induces hippocampal neuronal apoptosis by activating the c-Myc/chloride intracellular channel 4 (CLIC4) pathway [9]. Notably, the mitogen-activated protein kinase (MAPK) pathway is implicated in hippocampal apoptosis [10]. The MAPK family consists of stress-activated protein kinases (JNK), extracellular signal-regulated kinase (ERK) and p38 MAPK. The JNK signaling pathway has been reported to be involved in the apoptosis process of hippocampal neuronal regulated by oxidative stress [11]. ERK participates in the pathogenesis of children's learning disabilities by activating hippocampal apoptosis [12]. In addition, p38 MAPK have been demonstrated to mediate apoptosis via multiple mechanisms, such as increased c-Myc expression 
[13], Bax transposition, and caspase-3 activation [14]. However, whether the MAPK pathway mediates LPS-induced hippocampal apoptosis is unknown. Moreover, which member of the MAPK family is involved in the process of LPS-induced hippocampal apoptosis through the regulation of c-Myc/CLIC4 pathway is unknown.

Dexmedetomidine (DEX), as a neurological drug, possesses multiple biological activities such as antiinflammation [15], anti-oxidative stress [16] and anti-apoptosis [17]. Recently, it has been reported that DEX exerts neuroprotective efficacy in various brain injury models $[18,19]$. However, the specific underlying molecular mechanism is extremely complex and elusive. Importantly, DEX can reverse LPSinduced neuronal apoptosis [20]. Additionally, DEX has been proven to weaken apoptosis by inhibiting MAPK pathway activation [21]. DEX can also alleviate apoptosis through regulating p38 MAPK/ERK pathway and ROS/JNK pathway, respectively $[22,23]$. However, the detailed molecular mechanism of the protective effect of DEX on LPS-induced hippocampal apoptosis remains unclear.

Accordingly, in vivo, we focused on investigating the effects of DEX on MAPK family and c-Myc/CLIC4 pathway in LPS-induced hippocampal apoptosis. In vitro, further elucidate whether the p38 MAPK/cMyc/CLIC4 pathway is involved in the protective mechanism of DEX against LPS-induced hippocampal apoptosis by using the p38 MAPK inhibitor (SB203582).

\section{Materials And Methods}

\subsection{Animals and Treatments}

Forty-eight male Sprague Dawley rats ( 6 weeks old, 190-210g) were provided by the Central Hospital of Harbin Medical University (Harbin, China). Rats were maintained under standard conditions and access to water and standard rodent pellet food ad libitum [24]. All animal procedures in the present study were conducted according to the Animal Ethics Committee of the Northeast Agricultural University (SRM-11, China).

After a week of acclimatization, rats were divided at random into four groups $(n=12)$ : CON, LPS, LPS + DEX and DEX. LPS group was given LPS (Escherichia coli 0111: B4; Sigma-Aldrich, San Francisco, USA) dissolved in $0.9 \%$ physiological saline at $10 \mathrm{mg} / \mathrm{kg}$ by intraperitoneally (i.p.) injected. LPS + DEX group rats were i.p. with DEX (30 $\mu \mathrm{g} / \mathrm{kg}$, Wuhan Belka Biomedical Co., Ltd, Wuhan, China) $0.5 \mathrm{~h}$ before LPS administration. DEX group was treated with DEX (30 $\mu \mathrm{g} / \mathrm{kg}$, i.p.). In the CON group, rats were administered with an equal volume of physiological saline by i.p. injected. The doses of DEX and LPS refer to previous reports $[9,25,26]$.

\subsection{Sample Collection}

Four hours after the final treatment, all rats were anesthetized with isoflurane (Yipin Pharmaceutical Co., Ltd, Hebei, China) and sacrificed. Blood was quickly collected by cardiac puncture, and centrifuged at $3000 \mathrm{rpm}$ for $10 \mathrm{~min}$ at $4^{\circ} \mathrm{C}$ to take the supernatant for inspection of inflammatory indicators. The whole 
brains of three rats in each group were immediately fixed in $10 \%$ formalin solution for histopathological observation and immunohistochemical detection. Three hippocampal tissues per group were used for ultrastructural observation. The remaining four hippocampal tissues in each group were rapidly frozen in liquid nitrogen and then stored at $-80^{\circ} \mathrm{C}$ for Real-time PCR and Western blot analysis.

\subsection{Histopathology and Ultrastructural Observation}

Brain tissue samples fixed with $10 \%$ formalin for $24 \mathrm{~h}$ were dehydrated and then embedded in paraffin. Next, they were cut into 4-5 $\mu \mathrm{m}$ sections and stained with hematoxylin and eosin (H\&E, Wuhan Biotechnology Ltd. Co., Wuhan, China). After 5-10 min, all sections were observed and photographed under an optical microscope (TE2000, Nikon, Japan). The number of neurons in hippocampal CA1 and CA3 region was counted by ImageJ software (400x magnification).

After fixation with $3 \%$ glutaraldehyde for $48 \mathrm{~h}$, the hippocampus blocks $\left(1 \mathrm{~mm}^{3}\right)$ were exposed to $1 \%$ osmium tetroxide for $2 \mathrm{~h}$. Next, the blocks were dehydrated, embedded, sectioned ( $60 \mathrm{~nm})$, and then stained with lead citrate. All samples were captured by transmission electron microscope (Tecnai-G212, FEI Company, Netherlands). Based on the morphology and integrity of the mitochondrial membrane and cristae, the mitochondria in six discontinuous fields of each sample were scored. The criteria for judging mitochondrial damage are as follows: 0 , well-defined and organized membranes and cristae; 1 , minor distortions and/or swellings, but general organization retained; 2, major distortions and/or swellings and discontinuous membranes and cristae; 3 , membranes and cristae dissociated into particulates to produce diffuse mitochondrial ghosts; 4 , only a few mitochondrial remnants in cells.

\subsection{Immunohistochemistry Analysis}

Immunohistochemical analysis was performed as described previously [27]. Briefly, paraffin-embedded brain tissue slices were dewaxed with xylene, dehydrated with gradient alcohol, incubated with hydrogen peroxide, and sealed with goat serum. After that, they were incubated with primary and secondary antibodies and labeled with horseradish enzyme. DAB was used for color development. Finally, all slices were observed and photographed under a DP73 type microscope (OLYMPUS, Japan).

\subsection{Cell Culture and Drug Treatments}

PC12 cells were obtained from the Chinese Academy of Sciences (Shanghai, China) and cultured in DMEM medium (Gibco, Waltham, MA, USA) containing 10\% FBS and $1 \%$ penicillin-streptomycin in a humidified incubator $\left(37^{\circ} \mathrm{C}, 95 \%\right.$ relative humidity, $\left.5 \% \mathrm{CO} 2\right)$. The experimental groups were as follows: CON, LPS, LPS + DEX and LPS + SB. CON group: the cells were cultured in untreated medium. LPS group: the cells were cultured in medium supplemented with LPS. LPS + DEX group: the cells were treated with DEX for 30 min and then cultured in medium supplemented with LPS. LPS + SB group: after 30 min of p38 MAPK inhibitor SB203582 (10 $\mu$ M, Selleck.cn, Shanghai, China) administration, the cells were cultured in medium with LPS. The concentration of LPS and DEX was determined by CCK-8 assay.

\subsection{Cell Viability Assay and Observation of Cell Morphology}


Cells were seeded into 96-well plates $\left(4 \times 10^{3}\right.$ cells per well) for $12 \mathrm{~h}$, and then treated with/without DEX in the presence/absence of LPS. After $24 \mathrm{~h}$, the cell viability was determined with the CCK-8 kit (Beyotime Institute of Biotechnology, Suzhou, China) according to the manufacturer's instructions. The absorbance was read at $450 \mathrm{~nm}$ by a Bio-Tek Epoch microplate reader (Bio-Tek, Winooski, VT, USA). Cell morphology of each group was observed and captured by inverted microscope (Leica, Germany).

\subsection{LDH and ATP Release Assay}

Released LDH, ATP content and total ATPase activity were determined by LDH assay kit, ATP content assay kit and ultra-micro total ATPase kit (Nanjing Jiancheng Bioengineering Institute, Nanjing, China), respectively.

\subsection{Dual Immunofluorescence and Annexin V-FITC/PI Staining Assay}

Cells in each group were fixed with $4 \%$ paraformaldehyde for $30 \mathrm{~min}$ and then permeated with $0.3 \%$ Triton $\mathrm{X}-100$ for $10 \mathrm{~min}$. Next, they were incubated overnight with Bax (Wanlei, Shenyang, China) at $4^{\circ} \mathrm{C}$. The cells slides were incubated with CY3-labelled anti-rabbit lgG (Goodbio Technology, Wuhan, China) in the dark for $1.5 \mathrm{~h}$ at $37^{\circ} \mathrm{C}$, followed by washing 3 times with PBS. Afterwards, the cells were incubated overnight with Tom 20 (ABclonal, Wuhan, China) at $4^{\circ} \mathrm{C}$. Finally, they were incubated with 488 -anti-rabbit IgG (Goodbio Technology, Wuhan, China) in the dark for $1.5 \mathrm{~h}$ at $37^{\circ} \mathrm{C}$. Images were captured using a fluorescent inverted microscope (Nikon, Japan).

The resuspended cells were incubated with $5 \mu \mathrm{L}$ Annexin V-FITC and $10 \mu \mathrm{L}$ propidium iodide (Bioss, Beijing, China) for $15 \mathrm{~min}$. Then, cell apoptosis was detected by the Attune NxT flow cytometer (Thermo Fisher Scientific).

\subsection{Real-time PCR analysis}

Total RNA was isolated from hippocampus tissue and PC12 cells by using Total RNA Extraction Kit (Promega Biotech Co, Ltd, Beijing, China). According to the corresponding procedures, superscript II reverse transcriptase (Invitrogen, Carlsbad, CA, USA) was used for reverse transcription to obtain cDNA. Primer sequences of c-Myc, CLIC4 and GAPDH are shown in Table 1. RT-PCR analysis was performed using LightCycler480 (Roche, Basel, Switzerland). The relative quantification of the target gene expression was calculated according to $2^{-\Delta \Delta C t}$ method. 
Table 1

Primer sequence for real-time PCR detection.

\begin{tabular}{|c|c|c|}
\hline Gene & Accession number & Primer sequence $\left(5^{\prime}-3^{\prime}\right)$ \\
\hline \multirow[t]{2}{*}{ GAPDH } & \multirow[t]{2}{*}{ XM_216453 } & Forward: AGTGCCAGCCTCGTCTCATA \\
\hline & & Reverse: GATGGTGATGGGTTTCCCGT \\
\hline \multirow[t]{2}{*}{ c-Myc } & \multirow[t]{2}{*}{ NM_012603 } & Forward: GGAGAAACGAGCTGAAGCGTAG \\
\hline & & Reverse: CAGCCAAGGTTGTGAGGTTAGG \\
\hline \multirow[t]{2}{*}{ CLIC4 } & \multirow[t]{2}{*}{ NM_031818 } & Forward: GTCACCACCGTTGACCTGAA \\
\hline & & Reverse: TTGGGTGGGCACAAGACTTC \\
\hline
\end{tabular}

\subsection{Western blot analysis}

Hippocampal tissue and PC12 cells were lysed in RIPA buffer with PMSF and phosphatase inhibitor (Beyotime Biotechnology, Shanghai, China). After the protein concentration was determined using the BCA assay kit, the equivalent amount of protein samples was separated by SDS-PAGE gel electrophoresis, and transferred to the PVDF membrane. Then, membranes were blocked in $5 \%$ skim milk for $2 \mathrm{~h}$ at room temperature. Subsequently, membranes were incubated with primary antibodies adding Primary Antibody Dilution Buffer (Leagene Biotechnology, Beijing, China) overnight at $4^{\circ} \mathrm{C}$. The primary antibodies include Bax, cleaved caspase-3, cleaved caspase-9 (1:1000, Cell Signaling Technology, USA); Bcl-2, cytochrome C, P-JNK, JNK, P-ERK, ERK, P-p38, p38 (1:750, Wanlei, Shenyang, China); c-Myc, CLIC4 (1:1000, Santa Cruz Biotechnology Inc, USA). Next, they were incubated with appropriate combination of secondary antibody for $2 \mathrm{~h}$ at $37^{\circ} \mathrm{C}$. The immunoreactive protein bands were visualized using the enhanced chemiluminescence kit (Beyotime Biotechnology, Shanghai, China), and were captured by Amersham Imager 600 software (GE, USA). Finally, all protein bands were quantified with ImageJ software.

2.11. Statistical analysis

All data were represented as mean \pm SD and analyzed using IBM SPASS Statistics 23 software (SPASS, IL, USA). Statistical analysis was conducted via one-way ANOVA, followed by Tukey's post hoc test. Values with $P<0.05$ was considered statistically significant, $P<0.01$ were considered extremely significant.

\section{Results}

\subsection{DEX Improved LPS-induced Hippocampal Structural Lesion}


H\&E staining visually revealed the histopathological changes of hippocampus (Fig. $1 \mathrm{a}$ and b). In the CON and DEX groups, neurons in hippocampal CA1 and CA3 regions were arranged neatly and tightly, with round or oval nuclei and clearly visible nucleoli. In the LPS group, the neuronal arrangement was disordered, the intercellular space increased, nuclear pyknosis, nucleolus disappearance, partial cell lysis, and a large number of apoptotic and necrotic cells were observed. However, LPS-induced microstructural damage in hippocampal CA1 and CA3 regions was markedly ameliorated by DEX. Moreover, DEX significantly reversed the LPS-induced decrease in the number of neurons in hippocampal CA1 and CA3 regions $(P<0.01$, Fig. $1 \mathrm{c}$ and $\mathrm{d})$.

Ultrastructural observation results (Fig. 1f) showed that no abnormalities were captured in the mitochondria, nuclear membrane and other organelles of hippocampal neurons in the CON and DEX group. In contrast, most of the mitochondria in the LPS group were severely distorted and swollen, the mitochondrial membrane was discontinuous, the mitochondrial cristae dissolved and disappeared, and mitochondrial ghosts were occasionally seen. However, DEX treatment obviously reversed the aforementioned LPS-induced mitochondrial damage ( $P<0.01$, Fig. 1e).

\subsection{DEX Attenuated LPS-Induced Hippocampal Apoptosis via Mitochondrial Pathways}

As shown in Fig. 2, compared with the CON and DEX groups, the protein expression levels of Bax, Bax/Bcl-2, cytochrome C, cleaved caspase-9 and cleaved caspase-3 in the LPS group were markedly elevated $(P<0.01)$. Nevertheless, DEX effectively suppressed the upregulation of mitochondrial apoptosis-related protein expression $(P<0.01)$.

\subsection{Effects of DEX on the MAPK/c-Myc/CLIC4 Signaling Pathway}

To investigate whether the MAPK pathway mediated LPS-induced hippocampal apoptosis, JNK, ERK, and p38 protein expressions were examined (Fig. 3a). Interestingly, the protein expression levels of P-JNK (Fig. 3b), JNK (Fig. 3c), P-JNK/JNK (Fig. 3d), P-ERK (Fig. 3e), ERK (Fig. 3f), P-ERK/ERK (Fig. 3g) and p38 (Fig. 3i) in the LPS group were not significantly different compared with the CON group, except for P-p38 and P-p38/p38. Immunohistochemical staining showed that the expression of P-P38 protein in the LPS group was evidently higher than that in the CON group (Fig. 30). Notability, DEX effectively reversed these alterations wrought by LPS upon P-p38 and P-p38/p38 ( $P<0.01$, Fig. 3h and j). Moreover, DEX weakened the LPS-induced increase in protein $(P<0.05$, Fig. $3 \mathrm{k}$ and $\mathrm{I})$ and mRNA $(P<0.01$, Fig. $3 \mathrm{~m}$ and $\mathrm{n})$ expression levels of c-Myc and CLIC4.

\subsection{DEX Reduced LPS-induced PC12 cell Viability Inhibition, LDH and ATP Release}


After treating PC12 cells with gradient concentrations of LPS $(0,50,100,150,200,250,300,350$ and 400 $\mu \mathrm{g} / \mathrm{mL}$ ) for $24 \mathrm{~h}$, the cell viability was tested to screen the optimal drug concentration of LPS. As shown in Fig. $4 \mathrm{a}$, the cell viability of $250 \mu \mathrm{g} / \mathrm{mL}$ LPS was $52.54 \pm 5.031$, which was the suitable concentration for LPS modeling. Notably, to varying degrees, different concentrations of $\operatorname{DEX}(1,5,10,25,50,100$ and 200 $\mu \mathrm{M})$ attenuated the inhibition of PC12 cell viability induced by LPS $(250 \mu \mathrm{g} / \mathrm{mL}) .50 \mu \mathrm{M}$ DEX contributed to $\sim 100 \%$ viability and was therefore selected as the therapeutic drug concentration in this study (Fig. 4b).

The observation results of cell morphology (Fig. 4c) showed that the CON group cells were spindleshaped or polygonal with a large number. In contrast, the cells in the LPS group were round and the number was obviously reduced. After treatment with DEX and SB203582 (p38 MAPK inhibitor), the number of cells increased, most of them were fusiform or polygonal, and a few were round.

Compared with CON group, LDH level was significantly increased, ATP content and total ATPase activity were markedly decreased in LPS group $(P<0.01$, Fig. 4d-f). However, DEX and SB203582 dramatically reversed all these changes induced by LPS $(P<0.01)$.

\subsection{DEX Suppressed LPS-induced Activation of the p38 MAPK/c-Myc/CLIC4 Pathway in PC12 Cells}

The expression of P-p38, c-Myc and CLIC4 was markedly elevated by incubation with LPS, whereas pretreatment with DEX and SB203582 strongly blocked these increases $(P<0.01$, Fig. 5).

\subsection{DEX Ameliorated LPS-induced Apoptosis in PC12 Cells}

To authenticate that DEX attenuates LPS-induced apoptosis by regulating the p38 MAPK/c-Myc/CLIC4 signaling pathway, Bax and mitochondria co-localize, Annexin V-FITC/PI staining assay and mitochondrial apoptosis pathway-related protein expression detection were performed. As shown in Fig. 6, Bax and Tom20 positive cells in the LPS group were significantly more than those in the CON group. In contrast, these positive cells were obviously reduced in the LPS + DEX and LPS + SB groups.

Annexin V-FITC/PI staining assay was used to evaluate PC12 cell apoptosis. Incubation with LPS significantly increased the rate of apoptosis, which was remarkably reduced in the LPS + DEX and LPS + SB groups ( $P<0.01$, Fig. 7a and b). The protein expression of Bax, Bax/Bcl-2, cytochrome $\mathrm{C}$, cleaved caspase-9 and cleaved caspase-3 was significantly increased by incubation with LPS, whereas pretreatment with DEX and SB203582 effectively adjusted these alterations $(P<0.01$, Fig. 7c-i $)$.

\section{Discussion}

LPS-induced animal models are widely used to study $A D$ and depression. $A D$ and depression are both disorders characterized by hippocampal dysfunction [28]. The clinical symptoms of AD are memory disturbance, behavioral derangement and cognitive impairment [29]. Late-life depression has been 
reported to be related to cognitive impairment [30]. Notably, the hippocampus, especially the CA1 and CA3 regions, represents a vital neural control center for behavior, emotion, learning and memory, and cognition [31]. Alterations in hippocampal structure are implicated in cognitive decline [32]. Fenghui Guo et al. have demonstrated that DEX improves LPS-induced cognitive decline. In the current study, DEX ameliorated the LPS-induced hippocampal microstructural and ultrastructural lesion, presenting as the decreased necrotic neurons, increased neuron density in CA1 and CA3 regions and improved mitochondrial damage. Thus, $D E X$ may be a promising drug for the prevention and treatment of $A D$ and depression.

Most reports of $A D$ and depression simulated with LPS have focused on neuroinflammation. Therefore, the understanding of the pathogenesis of $A D$ and depression is limited, which hinders the development of effective therapeutic drugs without side effects. Our previous studies have demonstrated that apoptosis is involved in the pathological process of LPS-induced neurodegenerative diseases [9]. Nanhui Yu et al. revealed that inhibition of neuronal apoptosis can alleviate cognitive impairment in AD model mice [33]. In addition, reducing apoptosis can improve depression-like behavior in rats [34]. The current results revealed that DEX attenuated the apoptosis of hippocampal neurons induced by LPS, which may be a potential neuroprotective mechanism of DEX against $A D$ and depression.

Numerous studies have corroborated that the MAPK pathway is a major signal transduction pathway that regulates apoptosis [35]. Deviation from strictly controlled MAPK signaling pathways contributes to the driving of many human diseases, including AD [36]. The MAPK family of mammals consists of JNK, ERK and p38. It has been reported that JNK is an important potential therapeutic target for depression [37]. Suppression of JNK attenuated hippocampal neuronal apoptosis and improved depression-like behavior in mice [38]. In addition, dephosphorylation of ERK leads to neuronal apoptosis, which contributes to depression [39]. Recent studies have shown that LPS can promote the phosphorylation of JNK and ERK in a variety of models in vitro and in vivo $[40,41]$. Surprisingly, our results showed no significant increase in the protein expressions of P-JNK, JNK, P-ERK, and ERK in the hippocampus of rats treated with LPS. This may be related to different modeling methods. The p38 signaling pathway is susceptible to being triggered by various signals including LPS [42]. Activated p38 MAPK cascade can induce neuronal apoptosis and lead to hippocampal neuronal damage [43]. Consistent with previous reports, in the current study, phosphorylated p38 is activated and participates in the process of LPS-induced neuronal apoptosis. However, the non-phosphorylated protein of p38 did not change significantly. Notably, DEX pretreatment markedly inhibited the phosphorylation of p38. These results suggest that inhibition of $p 38$ phosphorylation may be a protective mechanism for DEX to improve LPS-induced hippocampal apoptosis.

Furthermore, we found that DEX inhibited the LPS-induced increase in the protein and mRNA expressions of c-Myc and CLIC4 in the rat hippocampus. Recent studies have reported that p38 can promote the activation of c-Myc [44]. c-Myc, an effective apoptosis inducer, binds to the CLIC4 promoter and contributes to the activation of its transcription [45]. CLIC4 is a key mediator of c-Myc inducing apoptosis. Inhibition of CLIC4 activation can suppress c-Myc-induced apoptosis under various stress conditions. Notably, the c-Myc/CLIC4 signaling pathway is involved in LPS-induced apoptosis of hippocampal 
neurons. Therefore, we guessed that DEX exerts neuroprotective and anti-apoptotic effects by regulating the p38/c-Myc/CLIC4 signal pathway. This hypothesis was confirmed by observing the effect of the p38 inhibitor SB203582 in LPS-treated PC12 cells. Like DEX, SB203582 significantly reduced the protein and mRNA expressions of C-Myc and CLIC4. Moreover, as assessed by flow cytometry, SB203582 protects PC12 cells from LPS-induced apoptosis. Overall, our findings revealed that the p38 MAPK/c-Myc/CLIC4 signaling pathway was involved in the neuroprotective mechanism of DEX against LPS-induced apoptosis.

CLIC4, enriched in mitochondrial outer membrane, is implicated in mitochondrial pathway apoptosis. Overexpression of CLIC4 decreased mitochondrial membrane potential, causing cytochrome $\mathrm{C}$ to be released into the cytoplasm, thereby activating caspase [46]. Studies have shown that CLIC4 and Bax synergistically induce apoptosis, but their direct physical interaction have not been detected [47]. Bax, a pro-apoptotic protein, is present in the cytoplasm as a monomer in an inactive state. Under stress conditions, Bax is activated and translocated to mitochondria, forming heterodimer with $\mathrm{Bcl} 2$, leading to the release of cytochrome $\mathrm{C}$. Thus, Bax/Bcl2 is regarded as a switch that triggers apoptosis [48]. Our results showed that DEX and SB203582 blocked the translocation of Bax to mitochondria and downregulated the protein expressions of Bax/Bcl2, cytochrome $\mathrm{C}$, cleaved caspase- 9 and cleaved caspase-3. Collectively, DEX inhibited LPS-induced apoptosis in the mitochondrial pathway by regulating p38 MAPK/c-Myc/CLIC4.

\section{Conclusion}

In summary, our results demonstrated that DEX inhibited the activation of LPS-induced p38 MAPK/cMyc/CLIC4 signaling pathway, down-regulated the expression of mitochondrial apoptosis pathwayrelated proteins, thereby reducing neuronal apoptosis. This study elucidated the neuroprotective mechanism of DEX in LPS-induced hippocampal injury from the perspective of anti-apoptosis, which provides support for the clinical application of DEX in the treatment of Alzheimer's disease and depression.

\section{Declarations}

Funding This study was supported by National Natural Science Foundation of China (Grant No. 31772806 and 31902337) and Heilongjiang Key Laboratory for Laboratory Animals and Comparative Medicine, China.

Conflicts of Interest No conflict of interest.

Data Availability All data used to support this study are included in the article.

Code Availability Not applicable. 
Ethics Approval All animal experiment protocols in this study were approved by the Animal Ethics Committee of Northeast Agricultural University (SRM-11, China).

Consent for Participate Not applicable.

Consent for Publication Not applicable.

Abbreviations AD, Alzheimer's disease; LPS, Lipopolysaccharide; DEX, Dexmedetomidine; CLIC4, Chloride intracellular channel 4; MAPK, Mitogen-activated protein kinase; JNK, Stress-activated protein kinases;ERK, extracellular signal-regulated kinase; H\&E, Hematoxylin and eosin.

\section{Authors' contributions}

Yongping Chen: Conceptualization, Methodology, Writing - original draft, Writing - review \& editing, Supervision, Resources, Funding acquisition, Project administration. Lin Li: Investigation, Visualization, Validation. Jiuyan Zhang: Data curation, Formal analysis, Software. Hailin Cui, Jiucheng Wang: Investigation, Visualization, Validation. Chuqiao Wang, Mingxian Shi: Data curation, Formal analysis, Software. Honggang Fan: Conceptualization, Methodology, Writing - original draft, Writing - review \& editing, Supervision, Resources, Funding acquisition, Project administration.

\section{Acknowledgements}

This work was supported by the National Natural Science Foundation of China Grant (grant number 31772806), the Natural Science Foundation of Heilongjiang Province Grant (grant number C2017022) and the Heilongjiang Key Laboratory for Laboratory Animals and Comparative Medicine.

\section{References}

1. 2016 Alzheimer's disease facts and figures. Alzheimer's \& dementia: the journal of the Alzheimer's Association, 2016. 12(4): p. 459-509

2. Lim GY et al (2018) Prevalence of Depression in the Community from 30 Countries between 1994 and 2014. Scientific reports 8(1):2861

3. Eshel N, Roiser JP (2010) Reward and punishment processing in depression. Biol Psychiatry 68(2):118-124

4. Neal M, Richardson JR (2018) Time to get Personal: A Framework for Personalized Targeting of Oxidative Stress in Neurotoxicity and Neurodegenerative Disease. Current opinion in toxicology 7:127-132

5. Barch DM et al (2019) Hippocampal volume and depression among young children. Psychiatry research Neuroimaging 288:21-28 
6. Selkoe DJ (2001) Alzheimer's disease: genes, proteins, and therapy. Physiological reviews 81(2):741-766

7. Zhao Y, Jaber V, Lukiw WJ (2017) Secretory Products of the Human GI Tract Microbiome and Their Potential Impact on Alzheimer's Disease (AD): Detection of Lipopolysaccharide (LPS) in AD Hippocampus. Front Cell Infect Microbiol 7:318

8. Zhang J-C, Yao W, Hashimoto K (2016) Brain-derived Neurotrophic Factor (BDNF)-TrkB Signaling in Inflammation-related Depression and Potential Therapeutic Targets. Current neuropharmacology 14(7):721-731

9. Li B et al (2020) Role of c-Myc/chloride intracellular channel 4 pathway in lipopolysaccharideinduced neurodegenerative diseases. Toxicology 429:152312

10. Gao $S$ et al (2019) Qingxin kaiqiao fang ameliorates memory impairment and inhibits apoptosis in APP/PS1 double transgenic mice through the MAPK pathway. Drug Des Devel Ther 13:459-475

11. Zhang $\mathrm{H}$ et al (2020) Lycopene ameliorates chronic stress-induced hippocampal injury and subsequent learning and memory dysfunction through inhibiting ROS/JNK signaling pathway in rats. Food chemical toxicology: an international journal published for the British Industrial Biological Research Association 145:111688

12. Yan B et al (2019) Combined use of vitamin $E$ and nimodipine ameliorates dibutyl phthalate-induced memory deficit and apoptosis in mice by inhibiting the ERK $1 / 2$ pathway, 368 . Toxicology and applied pharmacology

13. Shan Z-L et al (2017) Shikonin suppresses proliferation and induces apoptosis in human leukemia NB4 cells through modulation of MAPKs and c-Myc. Mol Med Rep 16(3):3055-3060

14. Nakagami $\mathrm{H}$ et al (2001) Phosphorylation of p38 mitogen-activated protein kinase downstream of bax-caspase-3 pathway leads to cell death induced by high D-glucose in human endothelial cells. Diabetes 50(6):1472-1481

15. Feng $X$ et al (2019) Dexmedetomidine ameliorates lipopolysaccharide-induced acute kidney injury in rats by inhibiting inflammation and oxidative stress via the GSK-3 $\beta /$ Nrf2 signaling pathway. Journal of cellular physiology 234(10):18994-19009

16. Sha J et al (2019) Dexmedetomidine attenuates lipopolysaccharide-induced liver oxidative stress and cell apoptosis in rats by increasing GSK-3beta/MKP-1/Nrf2 pathway activity via the alpha2 adrenergic receptor. Toxicol Appl Pharmacol 364:144-152

17. Zhang Y, Tan X, Xue L (2018) The alpha2-adrenoreceptor agonist dexmedetomidine protects against lipopolysaccharide-induced apoptosis via inhibition of gap junctions in lung fibroblasts. Biochem Biophys Res Commun 495(1):92-97

18. Sun Y-B et al (2019) Dexmedetomidine inhibits astrocyte pyroptosis and subsequently protects the brain in in vitro and in vivo models of sepsis. Cell death disease 10(3):167

19. Zhang Y et al., Dexmedetomidine Promotes Hippocampal Neurogenesis and Improves Spatial Learning and Memory in Neonatal Rats. Drug design, development and therapy, 2019. 13: p. 44394449 
20. Ning Q et al (2017) Neurodegenerative changes and neuroapoptosis induced by systemic lipopolysaccharide administration are reversed by dexmedetomidine treatment in mice. Neurological research 39(4):357-366

21. Wang Q et al (2017) Dexmedetomidine inhibits activation of the MAPK pathway and protects PC12 and NG108-15 cells from lidocaine-induced cytotoxicity at its maximum safe dose, 91 . Biomedicine $\&$ pharmacotherapy $=$ Biomedecine $\&$ pharmacotherapie, pp 162-166

22. Wang K, Zhu Y (2018) Dexmedetomidine protects against oxygen-glucose deprivation/reoxygenation injury-induced apoptosis via the p38 MAPK/ERK signalling pathway. J Int Med Res 46(2):675-686

23. Chen $\mathrm{Y}$ et al., Dexmedetomidine Ameliorates Acute Stress-Induced Kidney Injury by Attenuating Oxidative Stress and Apoptosis through Inhibition of the ROS/JNK Signaling Pathway. Oxid Med Cell Longev, 2018. 2018: p. 4035310

24. Abd E, Fattah MA et al (2021) Rice Bran Extract Protected against LPS-Induced Neuroinflammation in Mice through Targeting PPAR-y Nuclear Receptor. Mol Neurobiol 58(4):1504-1516

25. Chen $Y$ et al (2019) Dexmedetomidine protects against lipopolysaccharide-induced early acute kidney injury by inhibiting the iNOS/NO signaling pathway in rats. Nitric Oxide 85:1-9

26. Zhang $\mathrm{H}$ et al (2019) Dexmedetomidine ameliorates LPS induced acute lung injury via GSK3ß/STAT3-NF-KB signaling pathway in rats. Int Immunopharmacol 74:105717

27. Zhang Z et al (2021) Protective Effect of GM1 Attenuates Hippocampus and Cortex Apoptosis After Ketamine Exposure in Neonatal Rat via PI3K/AKT/GSK3 $\beta$ Pathway. Molecular neurobiology

28. Yagi S, Galea LAM (2019) Sex differences in hippocampal cognition and neurogenesis. Neuropsychopharmacology: official publication of the American College of Neuropsychopharmacology 44(1):200-213

29. Hsu W-Y et al (2015) Effects of noninvasive brain stimulation on cognitive function in healthy aging and Alzheimer's disease: a systematic review and meta-analysis. Neurobiol Aging 36(8):2348-2359

30. Linnemann C, Lang UE (2020) Pathways Connecting Late-Life Depression and Dementia. Front Pharmacol 11:279

31. Vidal-Sancho L et al (2020) Decreased Myocyte Enhancer Factor 2 Levels in the Hippocampus of Huntington's Disease Mice Are Related to Cognitive Dysfunction. Mol Neurobiol 57(11):4549-4562

32. Bettio LEB, Rajendran L, Gil-Mohapel J (2017) The effects of aging in the hippocampus and cognitive decline. Neurosci Biobehav Rev 79:66-86

33. Yu N et al., Ganoderma lucidum Triterpenoids (GLTS) Reduce Neuronal Apoptosis via Inhibition of ROCK Signal Pathway in APP/PS1 Transgenic Alzheimer's Disease Mice. Oxidative medicine and cellular longevity, 2020. 2020: p. 9894037

34. Fan C et al (2018) Neuroprotective Effects of Ginsenoside-Rg1 Against Depression-Like Behaviors via Suppressing Glial Activation, Synaptic Deficits, and Neuronal Apoptosis in Rats. Frontiers in immunology 9:2889 
35. Kim EK, Choi E-J (2015) Compromised MAPK signaling in human diseases: an update. Arch Toxicol 89(6):867-882

36. Kim EK, Choi E-J (2010) Pathological roles of MAPK signaling pathways in human diseases. Biochim Biophys Acta 1802(4):396-405

37. Hollos P, Marchisella F, Coffey ET, JNK Regulation of Depression and Anxiety. Brain plasticity (Amsterdam, Netherlands) (2018) 3(2): p. 145-155

38. Meng J, Wang D-M, Luo L-L (2019) CTRP3 acts as a novel regulator in depressive-like behavior associated inflammation and apoptosis by meditating p38 and JNK MAPK signaling, 120. Biomedicine \& pharmacotherapy = Biomedecine \& pharmacotherapie, p 109489

39. Li X et al (2016) PTPRR regulates ERK dephosphorylation in depression mice model. J Affect Disord 193:233-241

40. Xu M et al (2020) Polysaccharide from Schisandra chinensis acts via LRP-1 to reverse microglia activation through suppression of the NF-KB and MAPK signaling. J Ethnopharmacol 256:112798

41. Lim H-S et al., Bakuchiol Suppresses Inflammatory Responses Via the Downregulation of the p38 MAPK/ERK Signaling Pathway. International journal of molecular sciences, 2019. 20(14)

42. Fan M et al (2019) LPS Induces Preeclampsia-Like Phenotype in Rats and HTR8/SVneo Cells Dysfunction Through TLR4/p38 MAPK Pathway. Frontiers in physiology 10:1030

43. Dong Y, Kalueff AV, Song C, N-methyl-d-aspartate receptor-mediated calcium overload and endoplasmic reticulum stress are involved in interleukin-1beta-induced neuronal apoptosis in rat hippocampus. Journal of neuroimmunology, 2017. 307

44. Wu Q et al., Resveratrol attenuates diabetes-associated cell centrosome amplification via inhibiting the PKCa-p38 to c-myc/c-jun pathway. Acta biochimica et biophysica Sinica, 2020. 52(1): p. 72-83

45. Shiio Y et al (2006) Quantitative proteomic analysis of myc-induced apoptosis: a direct role for Myc induction of the mitochondrial chloride ion channel, mtCLIC/CLIC4. J Biol Chem 281(5):2750-2756

46. Suh KS et al (2004) The organellular chloride channel protein CLIC4/mtCLIC translocates to the nucleus in response to cellular stress and accelerates apoptosis. J Biol Chem 279(6):4632-4641

47. Fernández-Salas E et al., mtCLIC/CLIC4, an organellular chloride channel protein, is increased by DNA damage and participates in the apoptotic response to p53. Molecular and cellular biology, 2002. 22(11): p. 3610-3620

48. Hengartner MO (2000) The biochemistry of apoptosis. Nature 407(6805):770-776

\section{Figures}


a
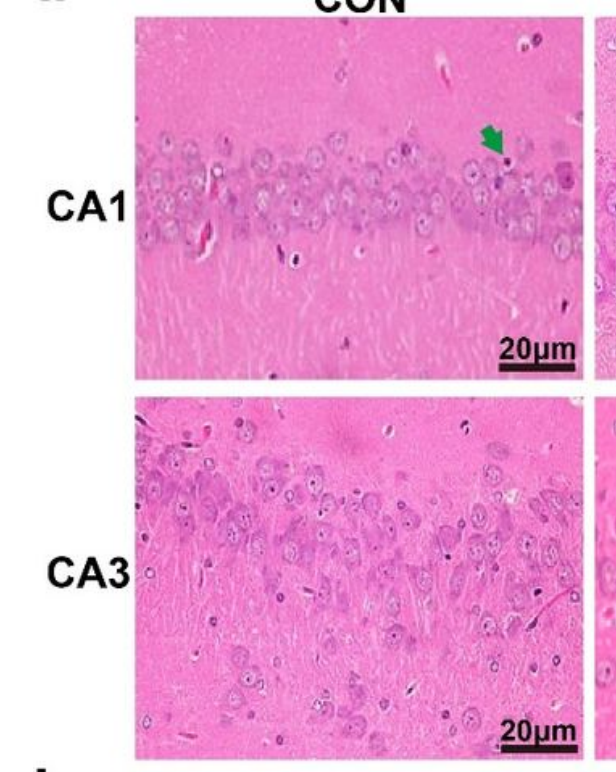

b

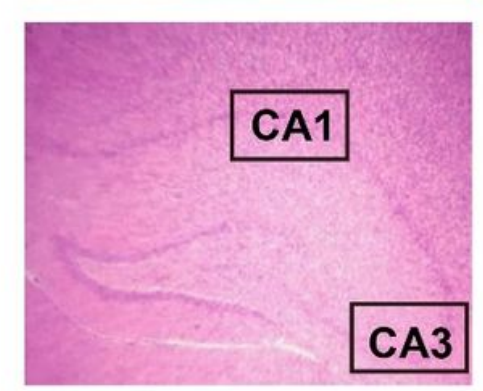

$\mathbf{f}$

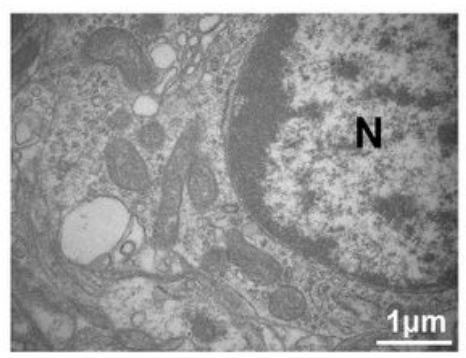

CON
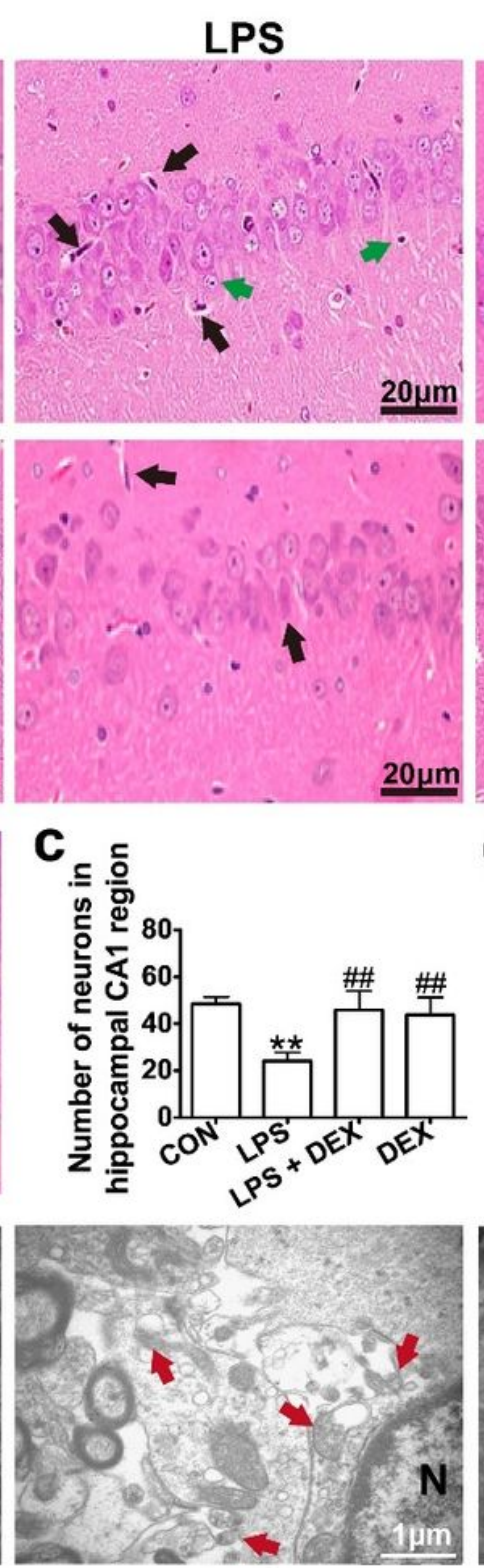

LPS
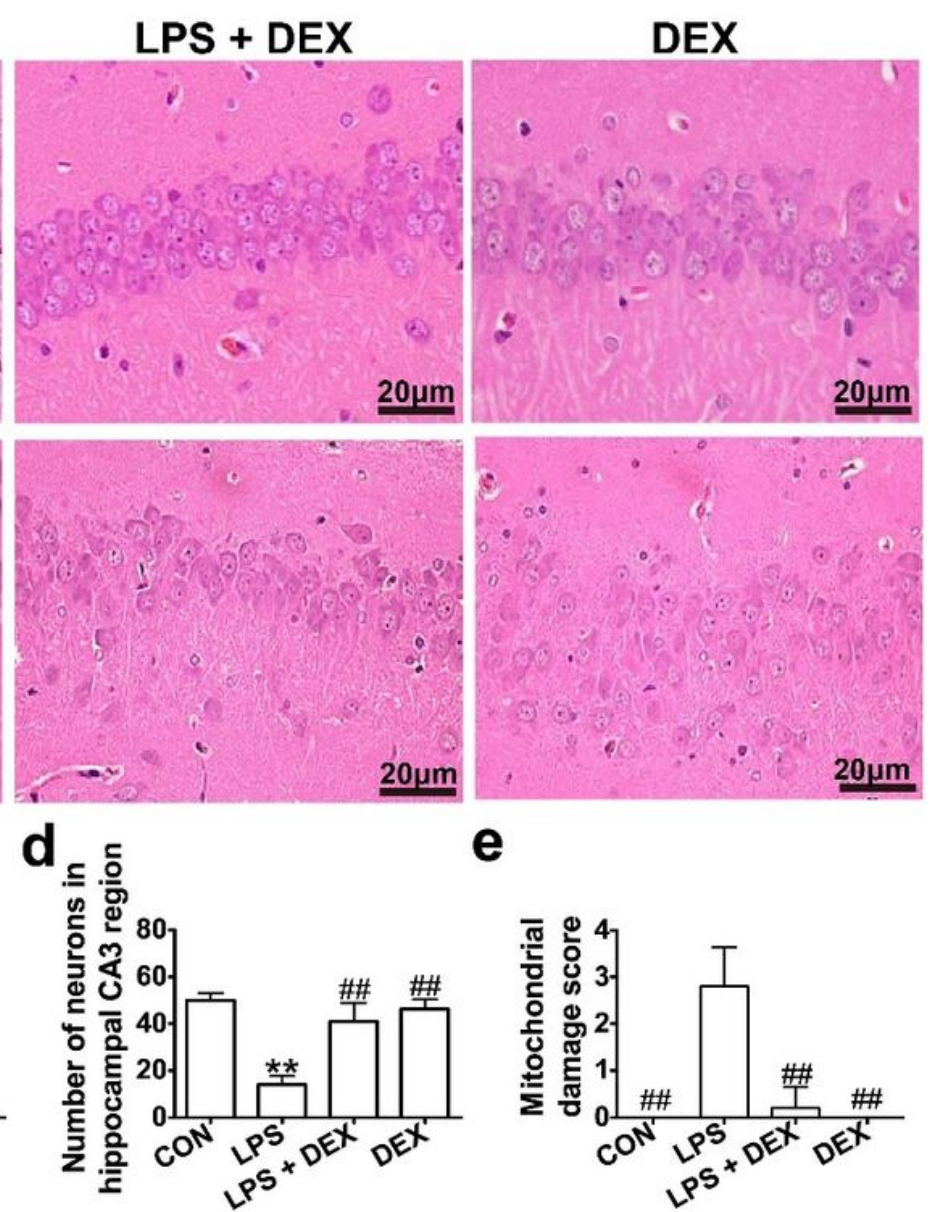

e

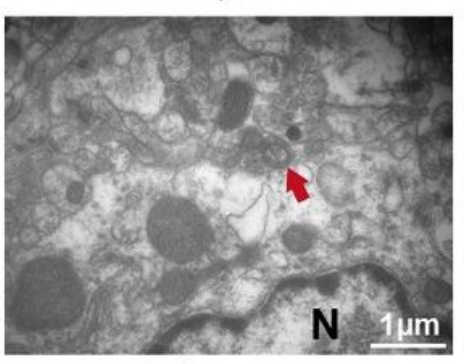

LPS + DEX
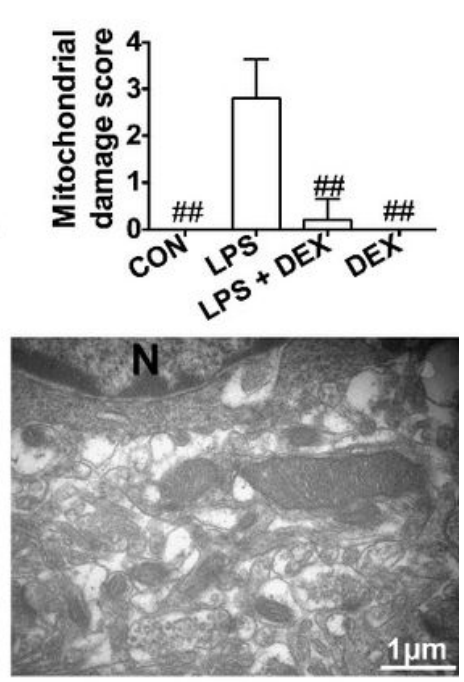

DEX

\section{Figure 1}

DEX improved LPS-induced hippocampal structural lesion. a Histopathological observation of hippocampal CA1 and CA3 regions in CON group, LPS group, LPS + DEX group, DEX group, (magnification 400x, scale bars $=20 \mu \mathrm{m}$ ). Black and green arrows indicate necrotic and apoptotic neurons, respectively. $\mathrm{b}$ The CA1 and CA3 regions of the hippocampus (magnification 40x, scale bars $=$ $200 \mu \mathrm{m})$. c Number of neurons in hippocampal CA1 region. $d$ Number of neurons in hippocampal CA3 region. e Mitochondrial damage score. $f$ Ultrastructural observation of hippocampal CA1 and CA3 regions. Red arrow indicates damaged mitochondria. Data were presented as mean $\pm S D(n=3)$. $* * P<$ 0.01 vs CON group. \#\#P $<0.01$ vs LPS group. 
a

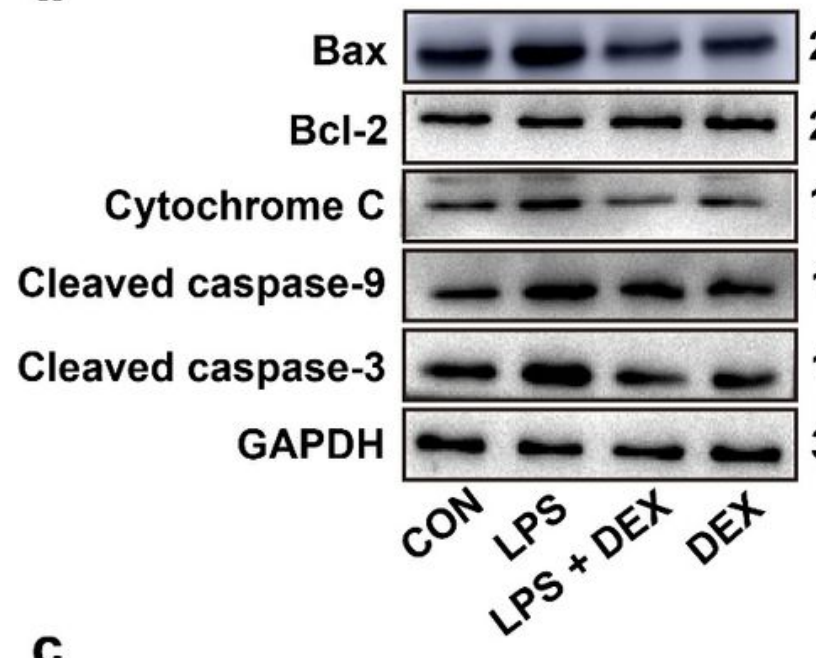

C

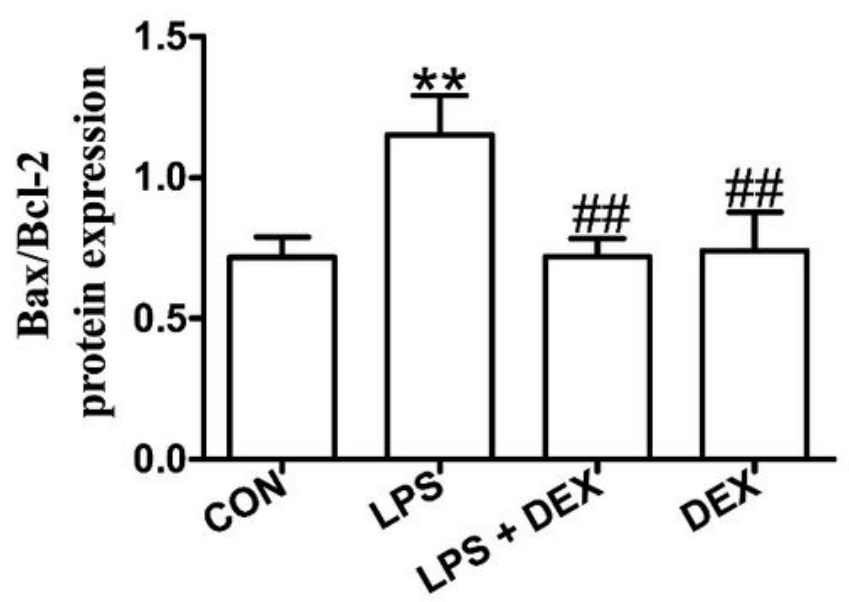

e

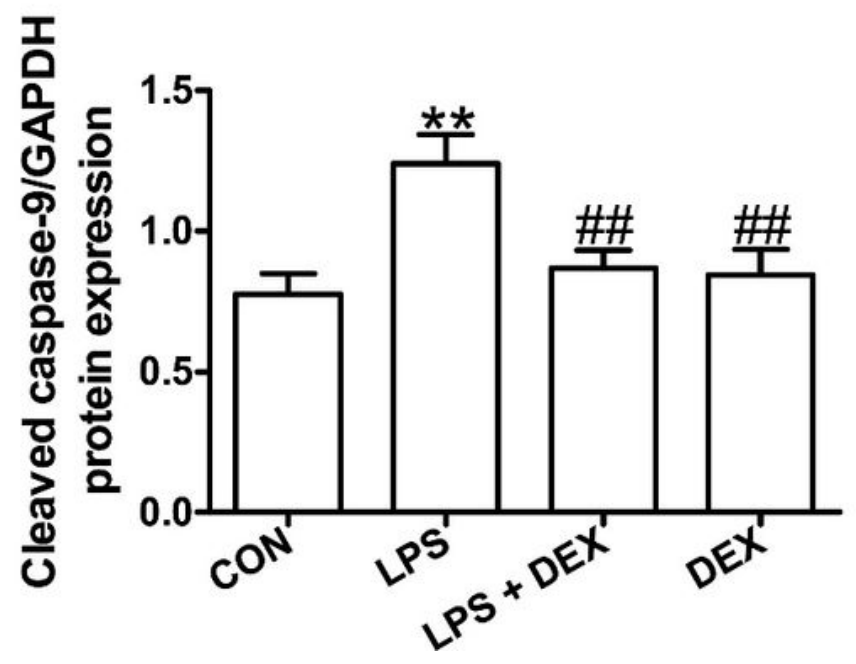

b

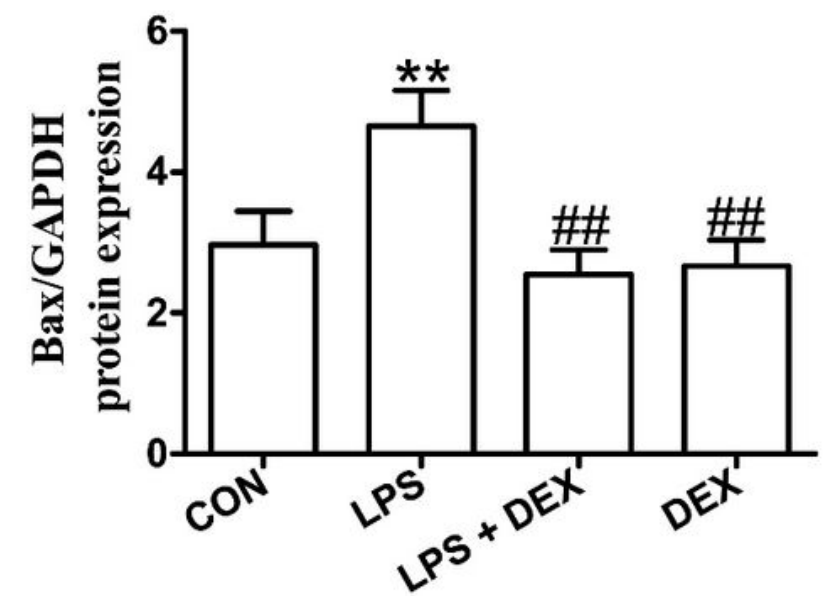

d

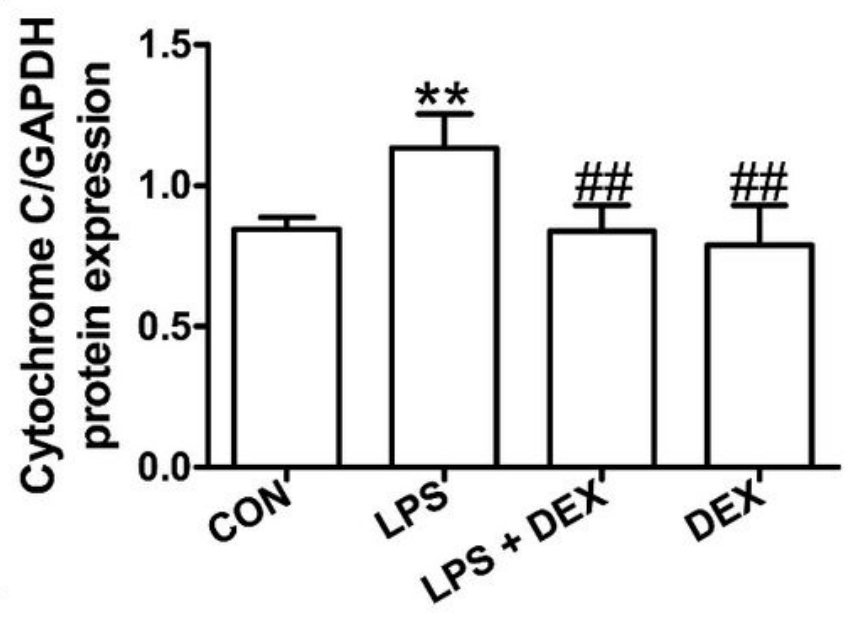

$\mathbf{f}$

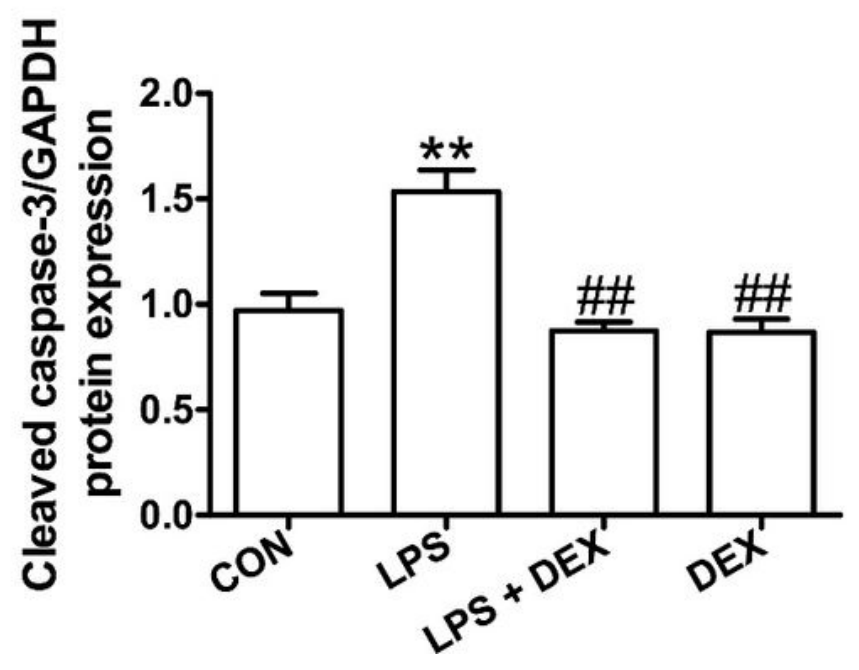

Figure 2

DEX attenuated LPS-induced apoptosis of hippocampal neurons via mitochondrial pathways. a Protein expression of Bax, Bcl-2, cytochrome $\mathrm{C}$, cleaved caspase-9 and cleaved caspase-3 in hippocampus. Protein quantitative analysis of (b) Bax, (c) Bax/Bcl-2, (d) cytochrome C, (e) cleaved caspase-9 and (f) cleaved caspase-3. Data were presented as mean $\pm S D(n=3)$. ${ }^{\star} P P<0.01$ vs CON group. \#\#P $<0.01$ vs LPS group. 


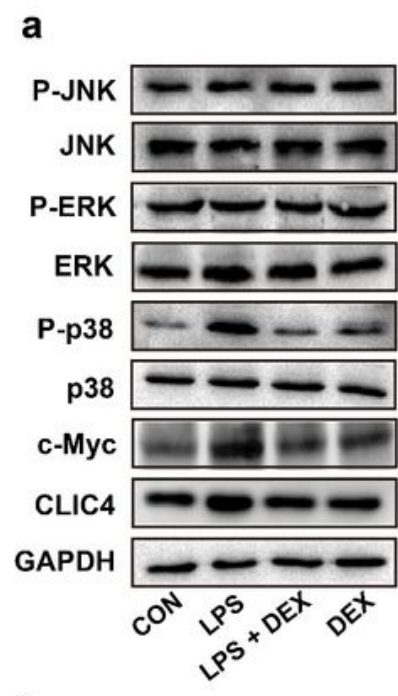

f

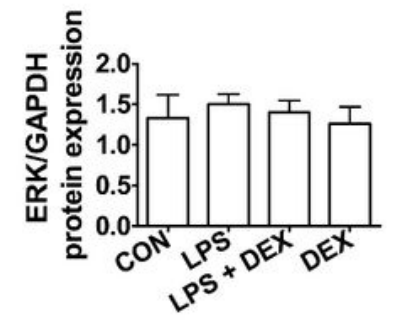

i

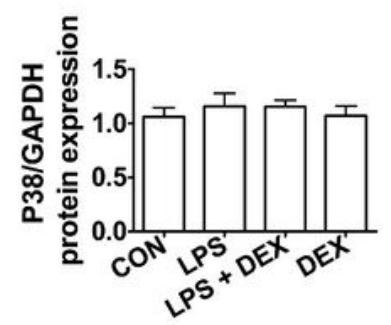

I

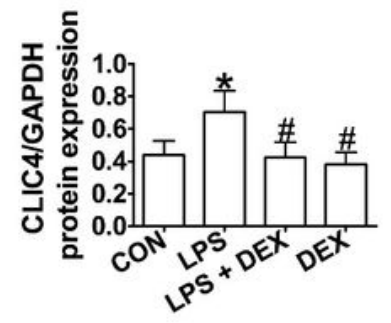

b

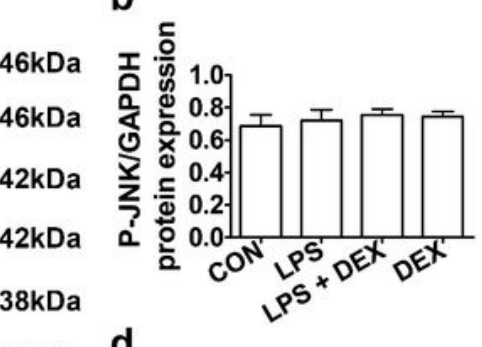

d

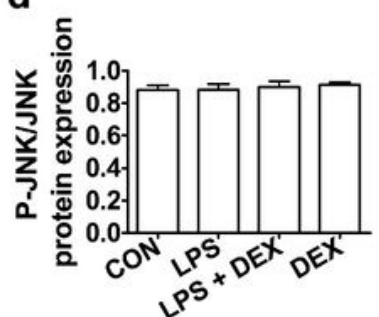

g

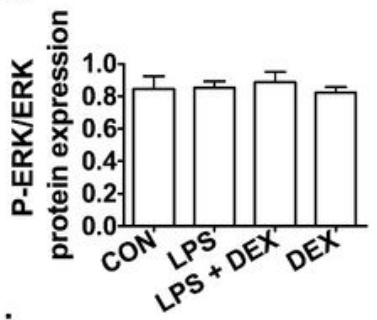

j

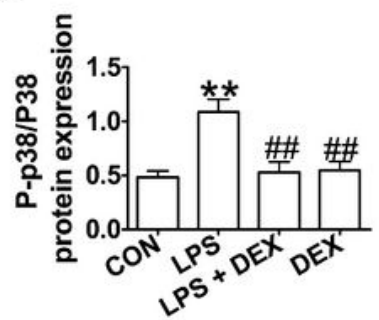

m

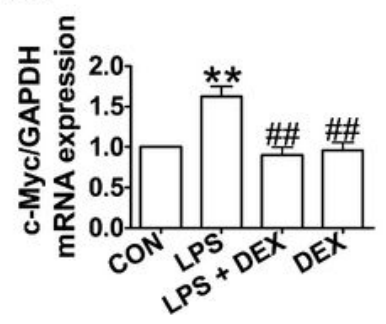

c

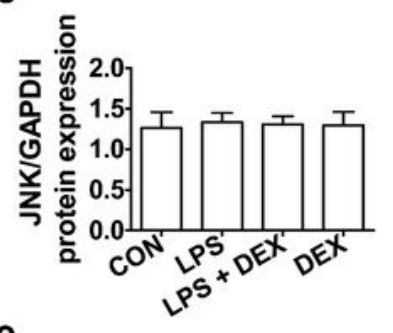

e

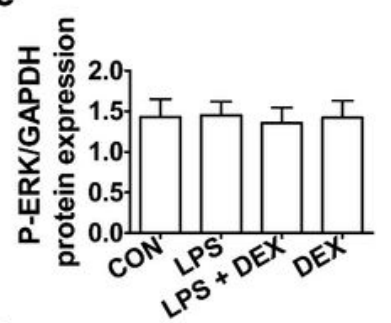

h

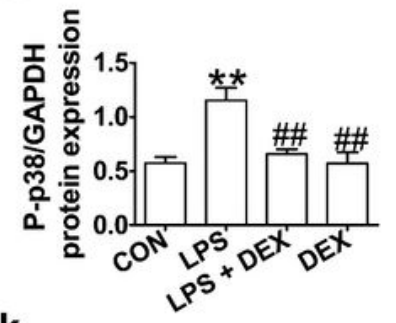

k

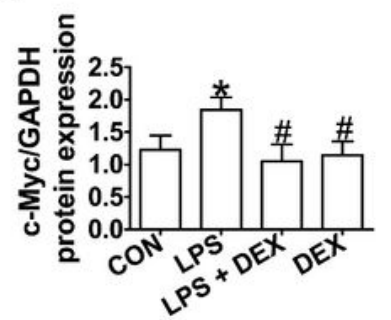

n

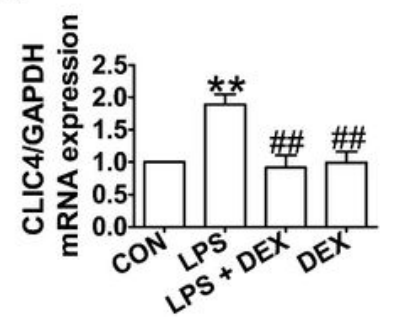

o

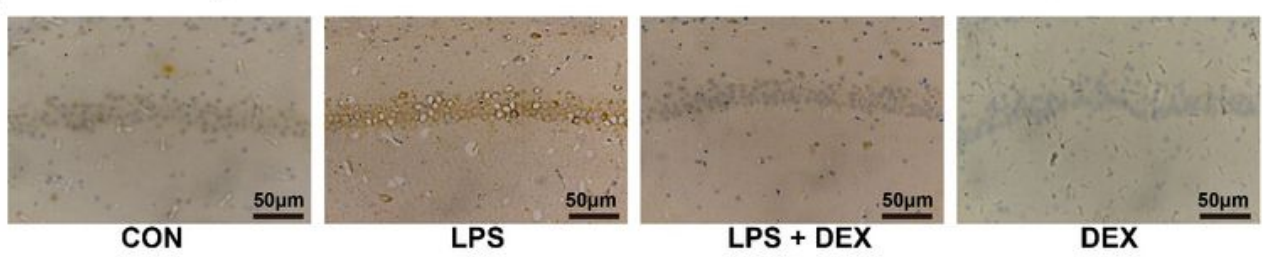

Figure 3

Effects of DEX on the MAPK/C-Myc/CLIC4 signaling pathway. a Protein expression of P-JNK, JNK, P-ERK, ERK P-p38, p38, c-Myc and CLIC4 in hippocampus. Protein quantitative analysis of (b) P-JNK, (c) JNK, (d) P-JNK/JNK, (e) P-ERK, (f) ERK, (g) P-ERK/ERK, (h) P-p38, (i) p38, (j) P-p38/p38, (k) c-Myc and (I) CLIC4. (m) c-Myc and (n) CLIC4 mRNA expression analysis. o Immunohistochemistry of P-p38 protein in the 
hippocampus (magnification 200x, scale bars $=50 \mu \mathrm{m}$ ). Data were presented as mean $\pm S D(n=3)$. *P< $0.05, * * P<0.01$ vs CON group. \#P $<0.05$, \#\#P $<0.01$ vs LPS group.

a

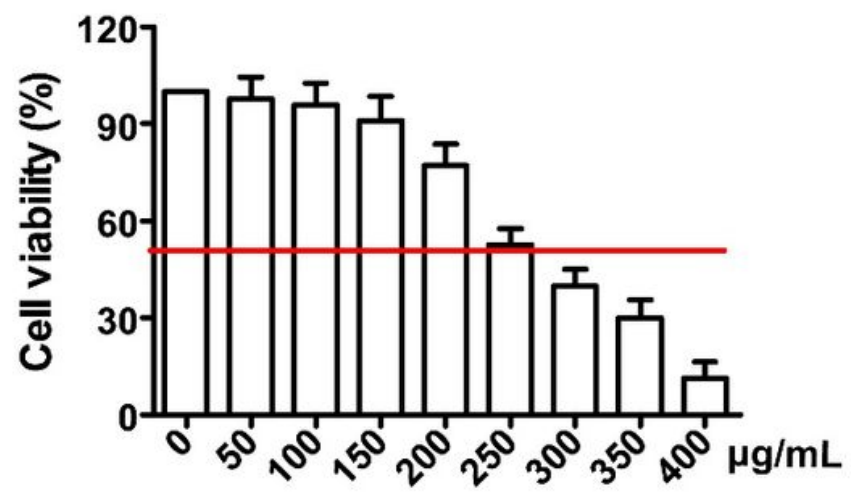

C

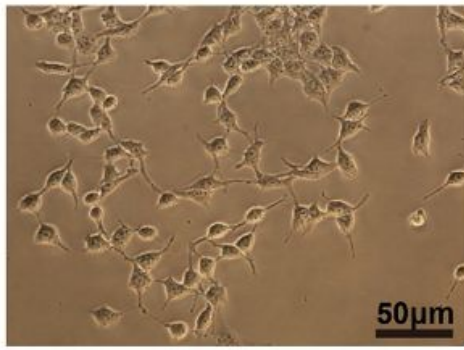

CON d

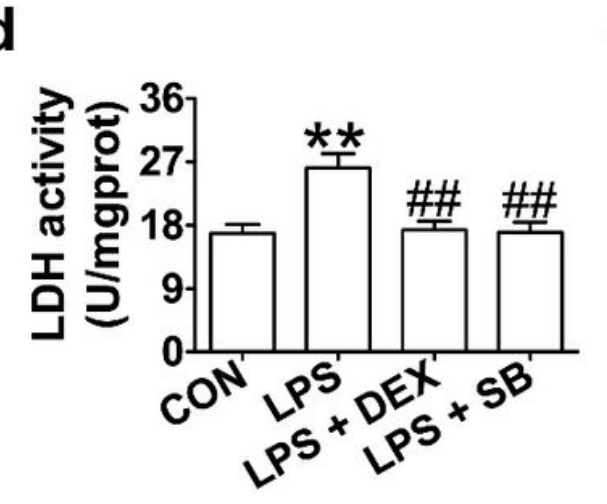

b

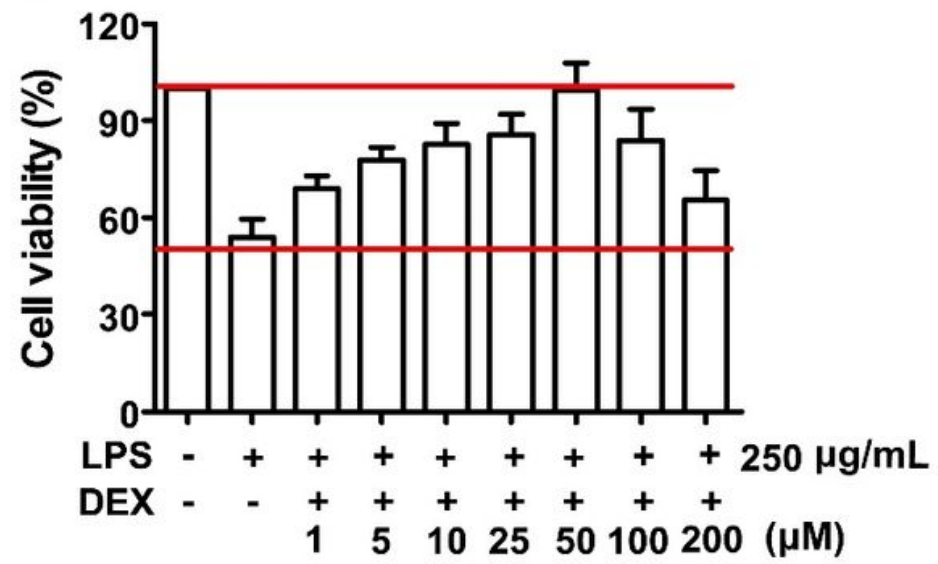

Figure 4

DEX reduced LPS-induced PC12 cell viability inhibition, $L D H$ and ATP release. a Cell viability of PC12 cells treated with different concentrations of LPS. b Cell viability of PC12 cells treated with different concentrations of DEX with or without LPS. c Cell morphology observation in the CON, LPS, LPS + DEX and LPS + SB groups, at 200x magnification, bars $=50 \mu \mathrm{m}$. d LDH activity. e ATP content. $\mathrm{f}$ Total ATPase activity. Data were presented as mean $\pm S D(n=6)$. ${ }^{\star \star P}<0.01$ vs CON group. \#\#P $<0.01$ vs LPS group. 
a

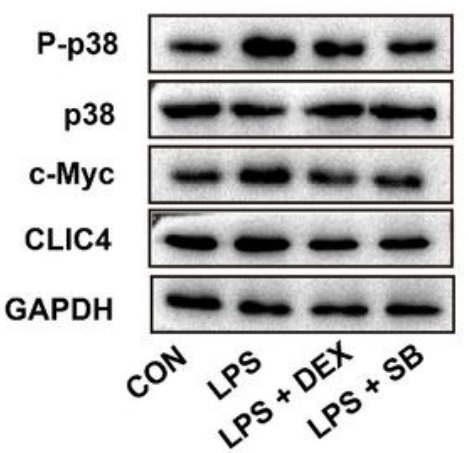

C

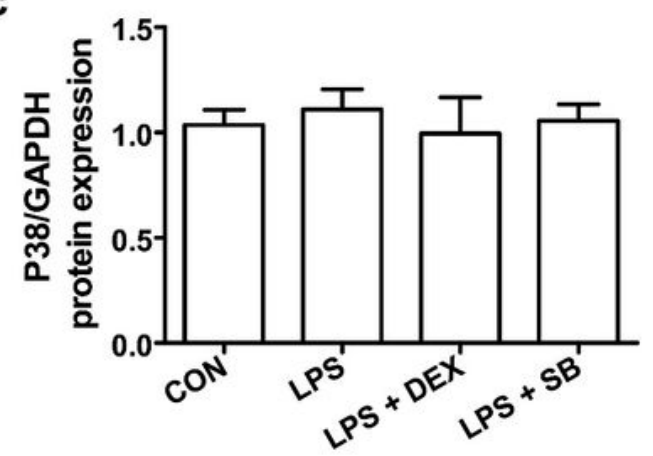

e

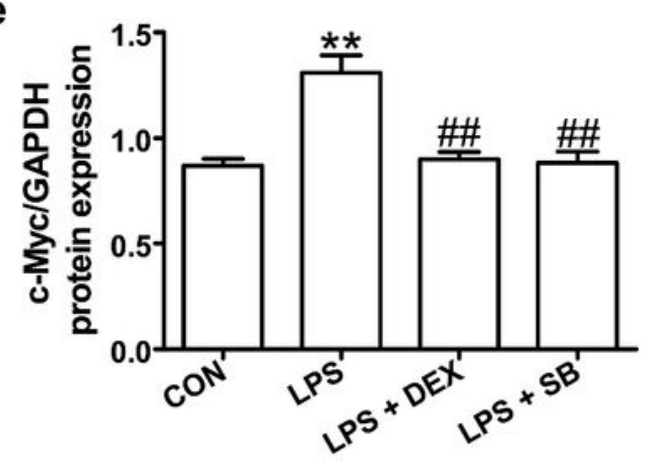

g

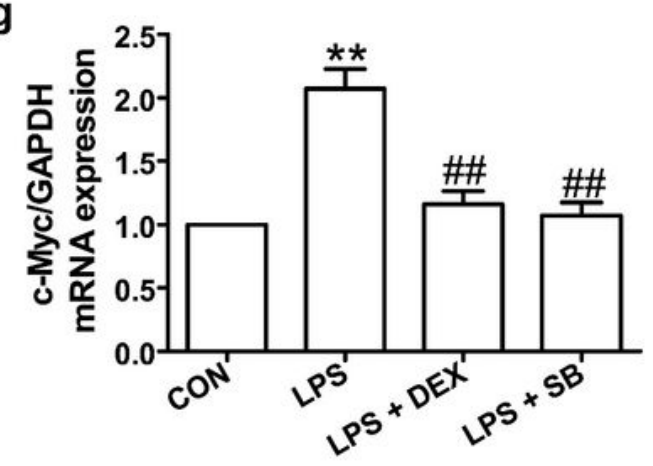

b

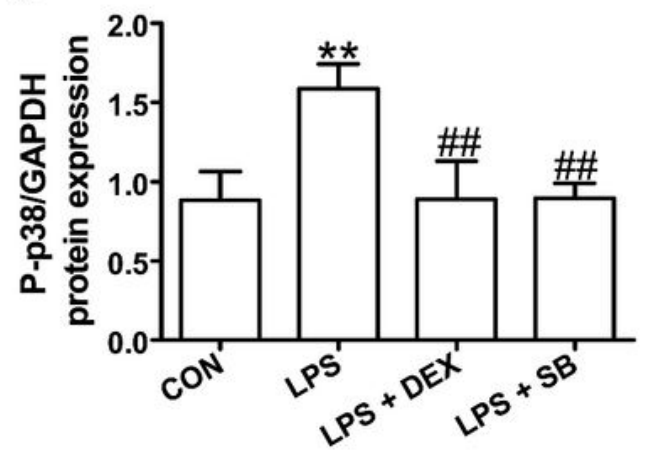

d

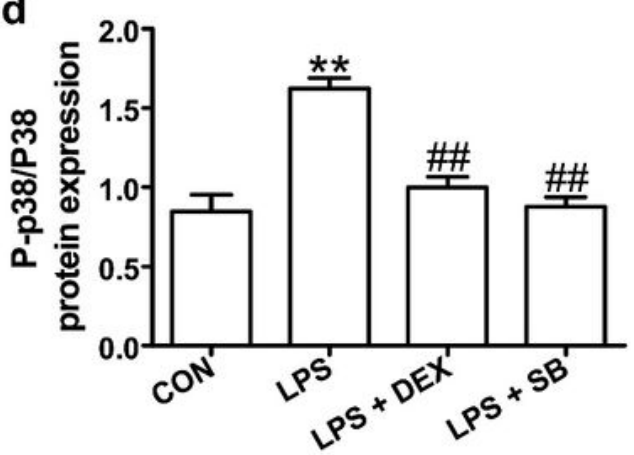

f

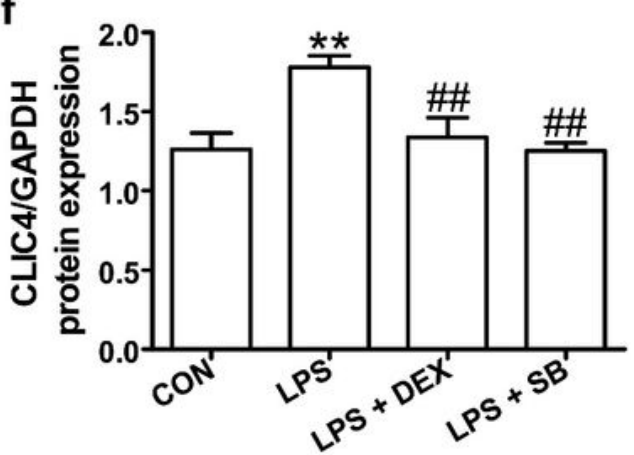

h

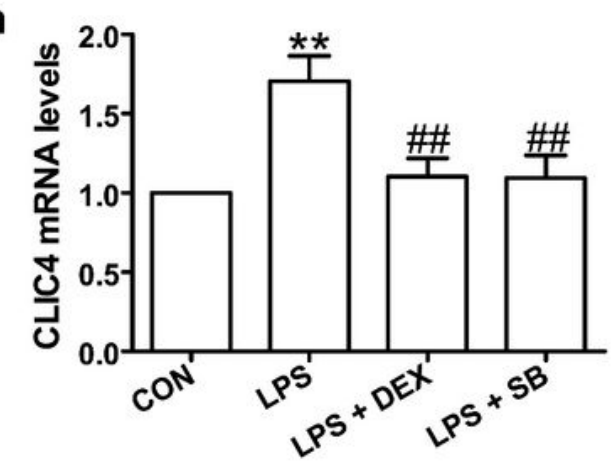

Figure 5

DEX suppressed LPS-induced activation of the p38 MAPK/c-Myc/CLIC4 pathway in PC12 Cells. a Protein expression of P-p38, p38, c-Myc and CLIC4. Protein quantitative analysis of (b) P-p38, (c) p38, (d) Pp38/p38, (e) c-Myc and (f) CLIC4. (g) c-Myc and (h) CLIC4 mRNA expression analysis. Data were presented as mean $\pm S D(n=3) .{ }^{\star * P}<0.01$ vs CON group. \#\#P<0.01 vs LPS group. 


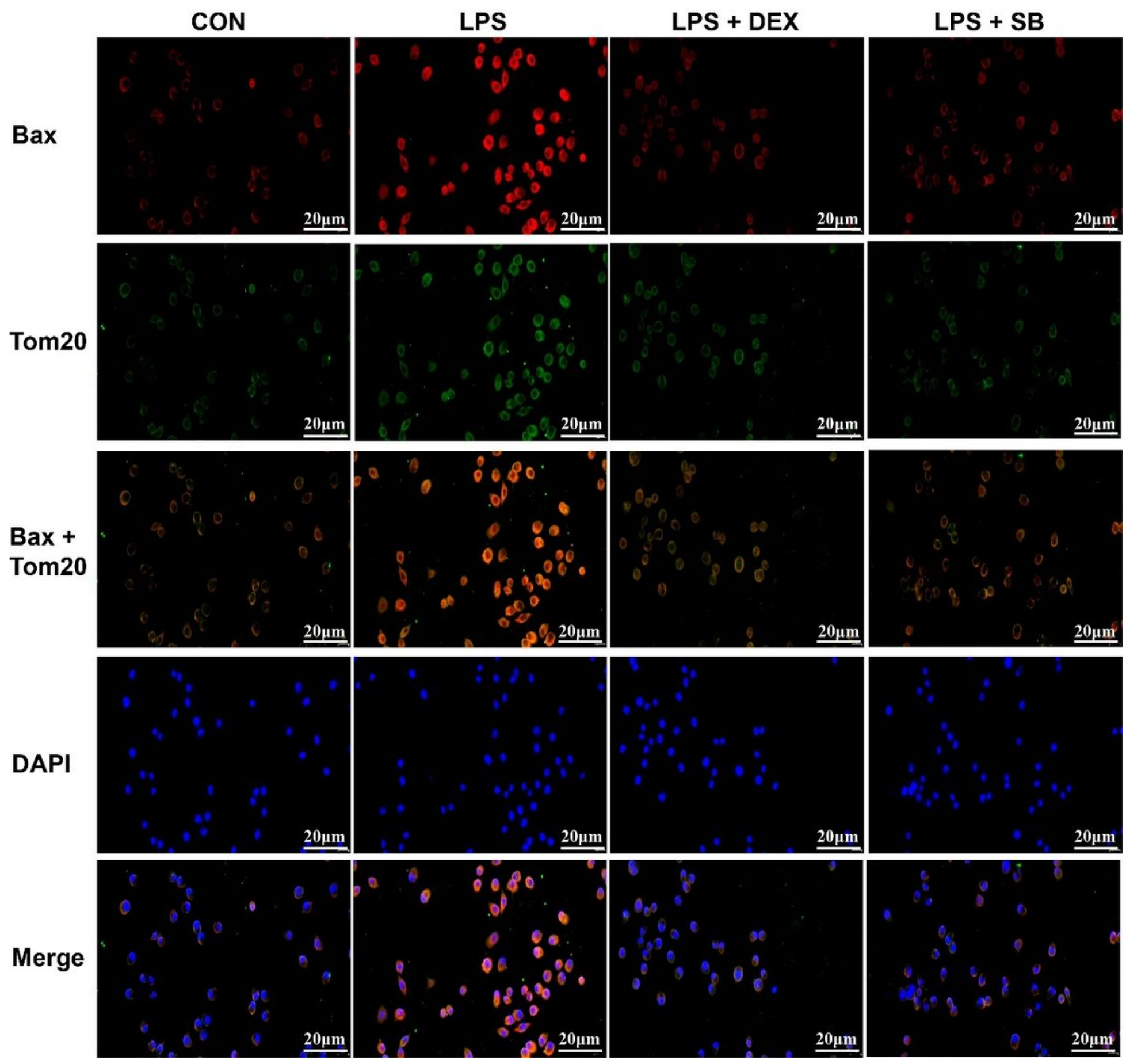

Figure 6

DEX inhibited the translocation of apoptotic protein Bax to mitochondria. The co-localization of Bax (red) and mitochondria (Tom20, green) was measured by double-labeled immunofluorescence assay $(n=3)$. Merge is the combination of Bax, Tom20 and DAPI (nucleus, blue). All microphotographs were observed and captured at $400 x$ magnification, bars $=20 \mu \mathrm{m}$. 
a

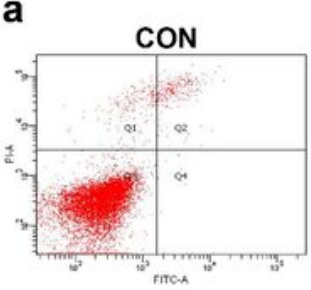

b

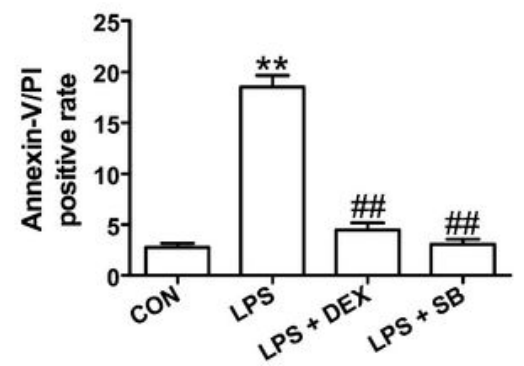

d

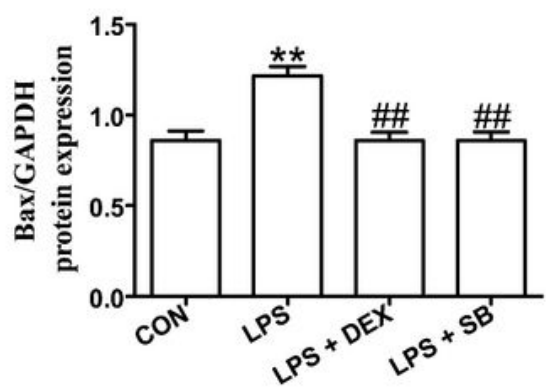

f

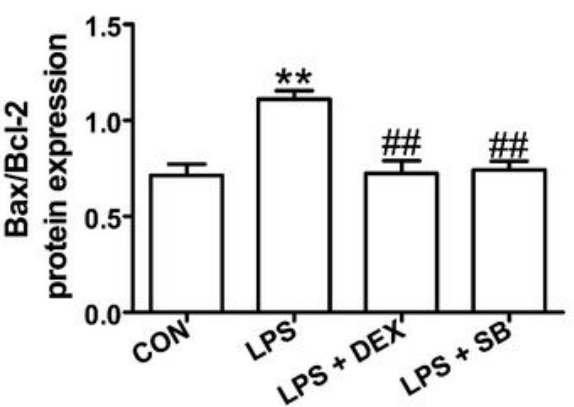

h

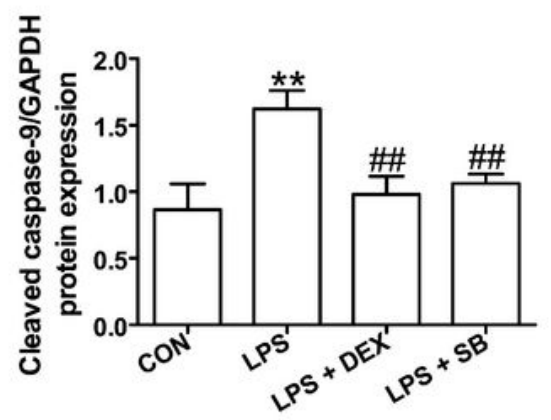

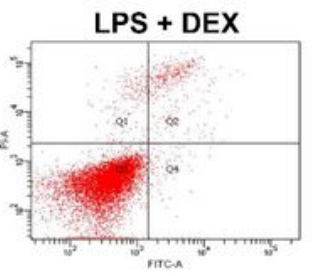
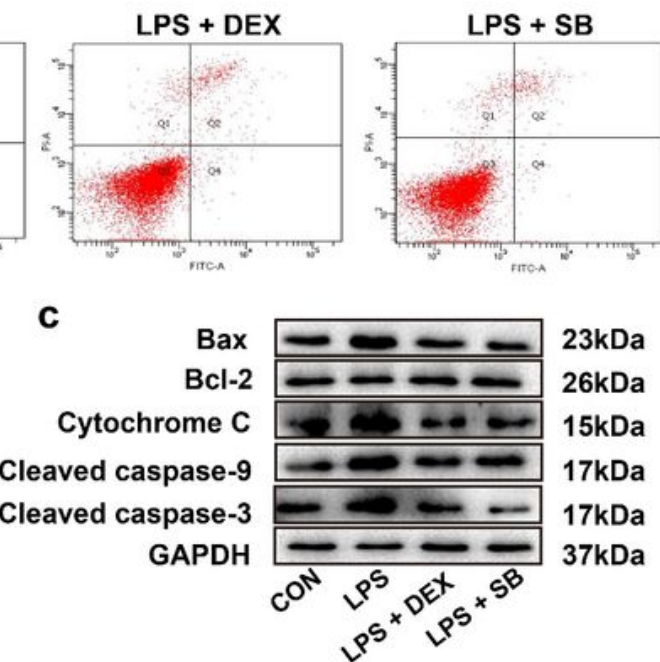

e

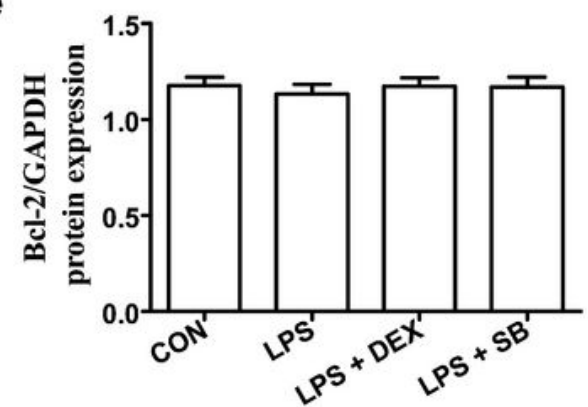

g

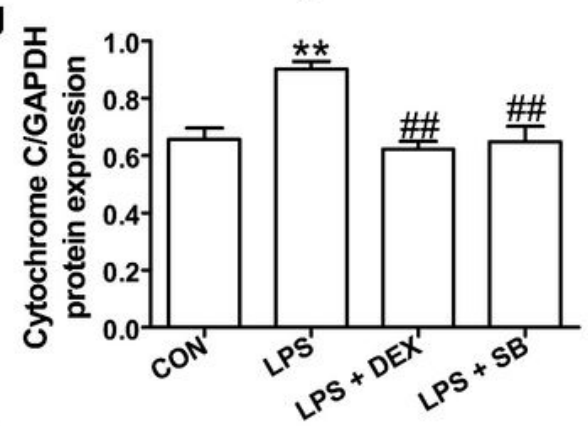

i

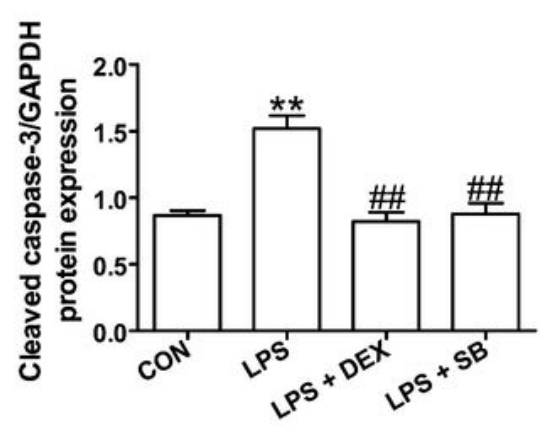

Figure 7

DEX ameliorated LPS-induced PC12 Cell apoptosis via the mitochondrial pathways. a PC12 cell apoptosis was detected by flow cytometry. b Annexin-V/PI positive rate. c Protein expression of Bax, Bcl2, cytochrome $\mathrm{C}$, cleaved caspase-9 and cleaved caspase-3. Protein quantitative analysis of (d) Bax, (e) $\mathrm{Bcl}-2$, (f) Bax/Bcl-2, (g) cytochrome C, (h) cleaved caspase-9 and (i) cleaved caspase-3. Data were presented as mean \pm SD $(n=3)$. ${ }^{*} P<0.01$ vs CON group. \#\#P $<0.01$ vs LPS group. 